\section{Talking about dying and death: a focus group study to explore a local community perspective}

\author{
Marilyn N. Y. Kirshbaum, ${ }^{1}$ Ian Carey, ${ }^{2}$ \\ Brigid Purcell, ${ }^{1}$ Seamus Nash ${ }^{3}$ \\ 'University of Huddersfield; ${ }^{2}$ Barnsley \\ Hospice; ${ }^{3}$ Kirkwood Hospice, UK
}

\section{Abstract}

There is a general perception held by health care practitioners based in hospices, palliative care services and general healthcare services that society is reluctant to talk about dying and death. This avoidance behaviour is observed, noted and expressed in national policy briefings as being detrimental to patient involvement in decision making, effective coping and preparation for death, organ donation, writing a will, and the process of bereavement. The aim of the pilot study was to explore the perceptions of a local community on the broad subject of Talking about Dying and Death. An interactive qualitative methodology using a constructivist approach enabled exploration of a wide range of views from a self-selected group resident within the local community $(n=8)$. Data were collected from a focus group session facilitated by the researcher and an associate researcher. Systematic and analytical coding of transcripts was undertaken using Framework Analysis (Richie and Spencer 1994). Four overriding themes were identified: i) emotions, beliefs and behaviours; ii) coping with adversity; iii) difficulties, barriers and tensions, and iv) fostering a participative future. There were some notions of superstition amongst the participants, but little mention of formal religious beliefs. Within the themes, Coping with Adversity and Difficulties, Barriers and Tensions is the core of the community's views and needs. The link to the local hospice service is significant for it is the place where practical help, spiritual care, and an appreciation for sensitivity, openness and honesty can be put into action. The importance of communication and language are critical above all else. Dying and death was articulated as an upsetting topic, and remains a taboo in this community in the United Kingdom, with a belief that talking will bring harm. Promotion of talking about dying and death was discussed in relation to the role of the local hospice and several suggestions were put forward.

\section{Introduction}

\section{Literature}

There is a general perception held by health care practitioners based in hospices, palliative care services and general healthcare services that society is reluctant to talk about dying and death. This avoidance behaviour is observed, noted and expressed in national policy briefings as being detrimental to patient involvement in decision making, effective coping and preparation for death, organ donation, writing a will and the process of bereavement. ${ }^{1}$ A collation of interested parties supported by the National Council for Palliative Care in the United Kingdom (UK) called Dying Matters has recently taken on the mantle to campaign for breaking down the barriers surrounding all aspects of dying and death in society, and to simply, talk more openly, easily and frequently about life's ultimate certainty. The coalition was established in 2008 with support from the Department of Health in the UK to raise the profile of end of life care and to change attitudes to death and dying in society (p. 11). ${ }^{1}$

The message that we all need to talk more about dying and death, might understandably sit slightly uncomfortably with most of us. However, from a health policy perspective, the task of encouraging communication and open discussion surrounding dying and death becomes a very real and complex challenge. How do well meaning, socially aware, community workers, educators, managers and health care practitioners integrate discussions about dying and death into their usual interactions with patients and clients? What are the different aspects to the topic of discussion? Is there a way to investigate what talking about dying and death actually means to both professionals and lay people in the community?

A preliminary literature search was undertaken to review previous relevant research using the search terms: death, dying, palliative care, end of life, talking and discussion within the online databases of Medline, CINAHL and PsychInfo. Despite the multitude of articles written about the philosophy and care of people at the end of life, there were only a small number of empirical studies that addressed the area of talking about dying and death.

Locke $^{2}$ suggests that talking about death and dying allows both the professional and the public to break the taboo and take a crucial first step towards ensuring good quality end of life care. In 2001, Gallagher ${ }^{3}$ held a public education event on death and dying to help raise the profile of palliative care within the community. The event, organised as a commercial trade show, furnished attendees with a questionnaire designed to assess their knowledge on issues about dying and their anticipated
Correspondence: Marilyn N. Y. Kirshbaum, University of Huddersfield, School of Human and Health Sciences, Queensgate (Ramsden Building 1/29), Huddersfield HD1 3DH, UK.

Tel. +44.0.1484.471277 - Fax: +44.0.1484.472380

E-mail: m.kirshbaum@hud.ac.uk

Key words: death, dying, palliative care, end-oflife, hospice.

Received for publication: 19 July 2011 Accepted for publication: 20 October 2011.

This work is licensed under a Creative Commons Attribution NonCommercial 3.0 License (CC BYNC 3.0).

(C)Copyright M.N.Y. Kirshbaum et al., 2011

Licensee PAGEPress, Italy

Nursing Reports 2011; 1:e8

doi:10.4081/nursrep.2011.e8

needs if they were to face a terminal illness (p. 52). ${ }^{3}$ The study reported that there was a need for both public and professional education around knowledge in the use of opioids within terminal illness. There were also strong views expressed concerning euthanasia. Attendees felt euthanasia involved withholding life sustaining treatments, and that patients should have free choice in their treatment. However, they did not necessarily agree with actions that would have death as a direct and immediate outcome. The Gallagher study concluded that educating the public is most valuable when public health oriented organisations reinforce the change through knowledge, individual attitudes and positive health behaviours. Furthermore, continuous education of health care practitioners and policy makers is also essential.

The potential contribution of general medical practitioners (GPs) was revealed in a project set in South Australia. ${ }^{4}$ A booklet was drafted for GPs to develop their confidence in broachingthe subject of death with their patients in addition to becoming more adaptable to changing perspectives of the public at large. Focus groups and interviews took place which involved of GPs working within palliative care teams and also GPs who expressed an interest in the project with an aim to improve the booklet. It was interesting to note, as the authors had, that the GPs suggested the inclusion of closed questions within consultations with patients. This contrasted sharply with the accepted view within the caring and counselling professions that open ended questioning should be used to explore an individual's views and perceptions. Furthermore the GPs expressed their view that few patients were able to face discussion about dying and death. However, until further research is conducted about what the public truly require and 
request, it will be difficult to change the practice of health care professionals.

A recent report by the Dying Matters Coalition $^{5}$ in the UK was published based on interim results from a study of GPs talking to patients about the subjects of death, dying and bereavement. Early conclusions were that: it is possible to increase GP confidence around talking about these issues; communication materials can assist in starting conversations; nine times out of ten a patient will continue a conversation about end of life when a GP starts one, regardless of whether the GP feels confident or not; and conversations between GP's, patients, family members, and carers resulted in a significant impact on the nature of a patient's death, such as support for their loved ones, their preferred place of care and whether or not they wish to be associated.

It is against this backdrop of research that our study attempts to expose dialogues with the public about death and dying for the purpose of deepening understanding of this important area. Furthermore, it is timely that continued research is undertaken to contribute to the current momentum driven by national policy and strategy.

\section{The local context}

Our study is situated in a local hospice in a moderate sized town in England known for its extensive coal mining resources, close knit communities, identifiable local accents, and historical market. The experience of the hospice is that they have always benefited from very generous support from local people via the donation of funds and local events, but that most people remain reluctant to engage in discussion about dying and death. In addition, it has been observed informally that many people are not fully aware about the range of care provided by the hospice and are noticeably reluctant to engage in discussion about end-of-life care.

In a genuine attempt to contribute to the national agenda within palliative and end-oflife care, the local hospice service on several occasions has attempted to encourage discussion in the course of evening talks with local celebrities. Nevertheless, the turnout from the local community had been a disappointment. To address this concern, a study was commissioned by the hospice in an attempt to promote a more open discussion about death and dying.

\section{Materials and Methods}

\section{Aim and objectives}

The aim of the study was to explore the perceptions of the general public on the broad subject of Talking about Dying and Death. The three objectives of this study were: i) to explore the perceptions of dying and death from the perspective of the general public; ii) to identify the barriers that might impede open discussion of dying and death within this community; and iii) to propose potential interventions to increase participation in their communication about dying and death.

\section{Design}

A qualitative methodology was planned to produce the data and insights required to address the complex and potentially sensitive nature of the objectives. A constructivist approach was selected as a vehicle to obtain meaning through conversation within the structure of a focus group. Within this methodological approach participants and facilitators agree to centre their thoughts and discussion on developing understanding of an identified issue. Focus groups can be used to generate hypotheses based on the perceptions and insights of group participants, ${ }^{6}$ which can later be tested in future studies using quantitative methods. Focus groups are also known to be particularly suited to discussions of a sensitive nature. ${ }^{7}$ This approach encourages group interaction, exploration and possible consensus or disagreement, and would be observable and recordable.

\section{Sample}

The sample for at least one focus group from the local community was planned to be self-selected from a large database of hospice contributors, contractors, associates, volunteers, and associates of all types. The database was the property of the hospice and used for the occasional mailings conducted by the hospice to invite people to public events, send out newsletters and assistance with fundraising. The names and details of people on the database were not passed over to the researchers; instead it was agreed that hospice staff would send out invitations to potential participants in this study. Out of over three hundred letters of invitation on university letterhead, only 10 people responded, which was sufficient for only one focus group and allowed for attrition. Purposive sampling was envisioned in selecting each group that varied in terms of age and gender, but this was not achieved because so few expressed interest in participating.

In terms of the process for informed consent, once someone expressed an interest to participate in the study, a detailed information sheet was posted back to them about the study's aims and procedures along with contact details of the researchers and the time and place of the focus group session. The study was approved by the university's ethics panel before participants were approached and data collection commenced. If the participants became upset, tearful or angry as dis- cussion was likely to expose raw emotions, the researcher and facilitator for the session were both experienced and compassionate nurses and considered very able in soothing most difficult situations. In addition, the meeting was held in a venue that included a receptionist who would be able to call emergency services, if required. These last points upheld responsibilities of the lead researcher to ensuring the safety and wellbeing for all who took part in the session.

\section{Data collection method}

The focus group was held in the early evening with a light buffet meal in a comfortable, informal, friendly and non-threatening environment within a meeting venue in the town centre. Contributions by participants were greatly appreciated as they were giving up their time and sharing personal views. Therefore it was important for the research team to ensure that these volunteers were at ease and not frightened in any way. The session was planned to be of sufficient length to ensure an exploration of the questions, and any subsidiary questions that could possibly arise from the participants. It was envisioned that 45-75 min should be adequate. The session was structured to begin with a warm up opening along with infusions of trigger objects that would help promote open and productive discussion (Table 1). The focus group was audio-taped with individual consent forms signed prior to data collection. The facilitator explained the purpose of the study, the format for the focus group and stressed that confidentiality and anonymity was to be respected amongst those in the room. Participants were also reminded that they could withdraw from the study at any time should they not want to continue.

A set of pre-determined questions guided the discussion. A short debriefing warm down exercise concluded the focus group session; at this time, the participants were encouraged to sit a moment and reflect upon the previous hour, their feelings, and any aspect of their experiences. An index card was passed to each participant to record their thoughts for themselves or for the benefit of the research. Several individuals produced summaries of the sessions while a few recorded quite personal and seemingly emotional recollections and thoughts that they wanted to share.

\section{Analysis}

The focus group session was digitally audiorecorded and transcribed by a paid member of the research team. Systematic and analytical coding of the transcripts generated themes which related to the study objectives. The five staged Framework Analysis described by Richie and Spencer $^{8}$ was followed: Familiarization, Identification of thematic cod- 
ing framework, Indexing, Charting and Mapping/Interpretation were used to guide the research team. All four researchers were included in the analysis process and communicated at set intervals to discuss the data and reflect upon emergent findings. Throughout the analysis process, the researchers planned to be open and cognizant of the possibility of emergent hypotheses that could result in further research and investigation.

\section{Familiarization}

Familiarization was achieved though listening to audio files, transcription, reading the transcription while simultaneously, or at a later stage, identification of interesting words, phrases, concepts, perceptions, similarities, and conflicts. This is an intuitive process within this familiarization and purely explorative phase.

\section{Identification of thematic coding framework}

Four conceptual themes with between 3-5 sub-themes each were identified from the focus group as displayed in Table 2.

Table 1. Format of focus groups and guiding questions.

1. Welcome, refreshments and introduction of facilitators

2. Review of purpose, ground rules and signing of consent forms

3. Warm up/ice breaker: What are your expectations for this focus group session?

4. Challenging assumptions: What are your specific views about talking openly [to whoever] about dying and death?

5. Who should be involved in the discussion?

6. What are the difficulties or barriers to talking about dying and death?

7. What could be done to promote more openness in discussion [and to inform future strategies?]

8. Warm down/debrief exercise using index cards to reflect on experience, reminder of confidentially, final thank you

Table 2. Thematic coding framework.

\begin{tabular}{ll} 
Themes & Subthemes \\
1. Emotions, beliefs and behaviours & $\begin{array}{l}\text { a) fear and worry } \\
\text { b) acceptance } \\
\text { c) influence of culture, faith and family }\end{array}$ \\
2. Coping with adversity & $\begin{array}{l}\text { a) practicalities } \\
\text { b) language } \\
\text { c) personal and family communication }\end{array}$ \\
\hline 3. Difficulties, barriers and tensions & a) family tensions \\
4. Fostering a participative future & b) medical language
\end{tabular}

reduction process must be conducted with care to ensure that credible data is not discarded. It is an issue of trustworthiness, validity and reliability of the entire methodological approach to ensure that the intended meaning of the participants is not misconstrued. All excerpts included identifier information regarding the focus group session and page number.

A table was developed and used as a template for the team to record pertinent, representative or particularly noteworthy examples (direct quotes) from each transcript along with the researchers own narrative observation. A summary of each theme and subtheme was then recorded.

\section{Findings}

Eight individuals attended the focus group session, which was organised by the sponsoring hospice. There were two men and six women in the group; the majority of them were judged to be over 60 ; however, demographic questions pertaining to age, marital status or level of education were not asked. The researchers only wanted to hear their views and were not intending to link any answers to a particular variable. The focus group lasted 90 min, significantly longer than had been planned. However, the group agreed to stay longer and expressed that they were committed to making their absolute and full contribution. In terms of participation, two women, an ex-nurse and a very articulate educated woman contributed substantially. It was noticeable that one man said very little throughout the session, except when he recounted a long, personal story. The other five participants contributed following slight encouragement from the facilitator. Many of their views, accounts and opinions featured in the transcripts and their excerpted quotations are presented below under corresponding themes.

Following the preliminary and subsequent in-depth and confirmatory analysis required for mapping and interpretation stage, four overriding themes identified included: i) emotions, beliefs and behaviours; ii) coping with adversity; iii) difficulties, barriers and tensions, and iv) fostering a participative future. These main themes and subthemes are presented as follows.

\section{Emotions, beliefs and behaviours}

\section{Fear and worry}

The taboo associated with talking about death either briefly or in a serious discussion was clearly evident. Several participants put forward the perspective that just talking about dying and death not only causes people to get upset, but also that the act of talking could also make death feel like a reality.

I think people would think if I talk about dying, or if I make a will, I am kind of wishing it on myself. [P1 p5]

It's like magic wishes. So if I talk about you dying, it's almost like am I wishing you dead. [P3 p5]

When mentioned to her mother that she was attending the focus group, a young woman recounted:

She said, don't get too upset will you. I think that's the fear...I think she's [mother] afraid that talking about it will make me upset, because I suspect talking about it makes her upset; which is why I can only do it in little bits. [P7 p28]

\section{Acceptance}

Acceptance that life is coming to an end and there is not much that can be done in terms of cure to prolong life was expressed overtly within the group. Inherent in the act of acceptance is an aspect that one must keeping going because things will get better, eventually. Across many cultures, rituals enable the person to either shield themselves from fear or accept difficult and upsetting situations. In this context, acceptance could provide the person with permission to get on and move forward, instead of dwelling upon fear or despair. 
Psychologically, the group alluded to the kind of mental tricks and avoidance games that were played. This example described a situation where the cancerous growth was referred to as an ulcer.

So I don't know whether I did right or wrong, playing that game with him. You know there were no money involved or owt [means 'anything' in local dialect], it were just that he just didn't want to know. So we just played this game and said he'd got an ulcer, you know and it went on for three years. [P5 p4]

However, it was also expressed that medical and nursing practice has changed over the years, so that:

Now they [health care practitioners]have to tell you, and we are not allowed to play the games we used to.[P5 p5]

The ethical principles of autonomy, justice and beneficence could be debated intensively on this last statement. However, here the comment contributed to our broad understanding within the theme of fear and worry, and provided a particular aspect of historical interest as it was noticed how the practice of withholding the truth of a cancer diagnosis from a patient was acceptable in less contemporary times.

\section{Influences of culture, faith and family}

Acceptance of dying and death was explored in greater depth in relation to family, faith, culture, and changing values. For example,

I think it's also to do with your belief systems; it has to do with whether you have a faith, for instance. [P4 p30]

Our family has always been quite open you know, and I was a nurse too, so that made a difference I think.[P5 p16]

Life and death are 'normal' therefore it is normal to discuss these types of issues [P4 p16]

And I think that's what's happening with the younger generation as well...there is no contact with the person that passes away and they might not even visit after the death. [P1 p11]

There was an interesting narrative of death and dying going full circle, in other words that life and death are normal, so therefore it is normal to discuss the many details and aspects of the process. Two of the participants were retired nurses and from their discourse it was noted that the professional could now make friends with the patient and that this was human, and not to be considered unprofessional. For example,

You build a strong relationship with your patient or your client. They may look at you as the professional but now sometimes you have got to draw the line...You become friends as well to a certain extent. [P5 p12]

I think that's changed over years actually $I$ remember being a pupil nurse and you weren't allowed to show absolutely any emotions anything, you had matron at the end of a ward. But people get upset if people die, and you are allowed to do that now. So I think in nursing that has changed I mean you haven't got to sit there blubbering. I appreciate that. But you can show that you are concerned and you do feel it and you are allowed to do that, but you weren't... years ago. [P5 p12]

\section{Coping with adversity}

\section{Practicalities}

There were benefits to having the practicalities of dying and death agreed and arranged in advance, in relation to end-of-life care, funeral arrangements, insurance, an invitation to a funeral, decision about a burial or cremation or type of service. However, for some, these discussions were fraught with intense emotion. Some people known to the participants would frankly refuse to think or discuss the inevitable.

The participants in this community group, by their very nature of volunteering to contribute to the discussion and attend as an evening activity, were forthcoming in sharing their own approaches.

In a way it can be helpful being busy making funeral arrangements immediately after a death. It kind of helps you get through that immediate phase. I mean when my dad died, which is ten years twelve years ago now. Although he and mum had talked about some things, like what music he wanted, it was very much down to her decisions about how she wanted the day to go. And she was in no state to make them, in the immediate aftermath of his death. So I was doing most of it for and with her. It was very difficult.[P2 p25]

When my mam passed away, my husband talked about burying and cremating. ..we went to look at me mums grave... he said, if you're going first you are going down there... So we bought plot. But as I'm getting older [sighs] You know it's that side of death I can't talk about the journey going down there [in the ground]. [P3 p22]

I have talked a little bit about it with my husband. We've not sat down and had a big discussion but when Coronation Street [television soap operal was on last week and it was Blanche's funeral and it said MOTHER [in flowers on top of the coffin], he turned to me and he said when I die you won't have some flowers in the shape of dad will you. And things like if it a piece of music comes on that he particularly likes, oh I would like this played at my funeral. That sort of thing...That's how you have to slip it in isn't it. [P4 p20]

We have talked about being cremated and as you say music, but trouble is it changes over life. I can remember saying some years ago that I like Elvis Presley [laugher] and then you change as you get older. [P1 p22]

\section{Language}

It was not surprising that it was observed by the community members that the words $d y$ ing and death were frequently avoided in favour of passing or passing away. Below are some excerpts from a lively phase of the focus group session.

Just going back to it being a taboo - I think it is, it's the new taboo, whereas I think sex used to be. Now it's death and dying even to the extent that now in the media you do not hear the word death, it's passed away, or passed, it's coming over from America. [P5 p6]

You just don't say that somebody has died anymore. Not like people used to. [P1 p6]

I notice it when they are bringing bodies back from the war [Afghanistan] they always talk about the brave solders coming home, not we are bringing their bodies back... So, you know the people stand and clap because our lads are coming home, we have brought them back. Actually they are dead. [P4 p7]

\section{Personal and family communication}

Children's issues and involvement were mentioned several times, e.g. Is death something that happens when someone is old? Where do they [dead people] go? It was obvious that all families are different even within a small, fairly stable and indigenous community. It was noteworthy that so many participants talked about estranged families.

I think it's good, you should tell your children, they should know about dying and death, and then it doesn't hit them as [badly]. I mean our Sophie she's only five. She said to me mam, 'Are you going to talk to me granddad?' And she said 'Why, where is he.' She said, 'He is there he's sat on settee-Aren't you going to talk to him?' And then the day after he died when they come and took his bed out of living room and that she were saying to - me granddad's gone to live up in sky and I am not going to be able to see him anymore. Then she turned round and said to him 'And you are going to die soon aren't you'? Cos he were an oldish person. [smiles] [P3 p12-13]

I mean I know from all the reading I have done about hospices, and about particularly about children who are facing death that they are very clear usually that they know what's happening, and they don't want people telling them stories about it.[P8 p15]

Families seemed to vary in terms of their level of comfort and practice when it came to talking about death and dying and communication skills of professionals were considered to be important, but the place of cultural norms and personal values seemed to play a significant role in this theme and be of interest for future research. 


\section{Difficulties barriers and tensions}

\section{Family tensions}

The families enveloped a particular form of social dynamic where protective mechanisms were used by both the relatives and the dying person; in other words, examples of collusion were revealed. For example:

My mother-in-law and I knew he Ihad cancer] but didn't want my father-in-law [the patient] to know. But my father-in-law said to me, I know I have got cancer and I know I'm dying - but don't tell Helen'.[P5 p11]

When the families used avoidance strategies, they did so because they thought it was beneficial to their loved one; however, what it actually did was to prevent honest and helpful discussion to take place about dying and death. This was substantiated by existent views that open discussion would take away hope and provided a feeling of giving in to disease and ill fate.

\section{Medical language}

There was a heated discussion about an almost universal view that the language used by health professionals was not as it should be. It was noted that either the language contained too much jargon and was not understandable, or that it was patronising and a far too basic interpretations of their medical condition.

I think they [health professionals] hide behind the language... they will try and kind of use a scientific word either that or they try and baby you. [P4 p32]

I was rushed into hospital recently with an acute attack of pain from my gall bladder, and its agonising pain. It was screaming, literally and the doctor who came to me said 'having a bit of trouble with your tummy then?' And honestly I wanted to hit him. 'No I have agonising pain in my abdomen!'[P4 p32]

This last example was more than just an annoyance, but could have an effect on diagnosis as the description of symptoms might be too simplified and not be communicated appropriately. Furthermore, it was noted that the inexperience of some health care practitioners was of concern as they missed cues from their patients. In the realms of talking about dying and death, this referred to avoidance strategies and poor communication skills, which were unfavourable qualities for a health care practitioner, particularly in the end-of-life care.

\section{Fostering a participative future}

\section{Outreach to the community}

Promotion of talking about dying and death was discussed in relation to the role of the local hospice service. Suggestions included: i) hosting varied events and discussion on all different topics of interest and not just centred on fundraising; ii) producing leaflets that provide practical information such as how to plan a funeral, useful assistance in the community; iii) hosting meetings with general medical practitioners to increase their awareness; iv) creatively thinking about how to publicise that the hospice is there to help and provide information about what it does (or could do) aside from providing palliative care; and v) opening up the hospice to the public and offering visitors a warm welcome in a pleasant environment was mentioned. In addition, beauty and complementary therapies could be offered to the public.

In the participants' words, this was expressed in terms of addressing the relationship between staff who are based in acute care hospitals and those within the specialist hospice service.

Maybe the hospice professionals could have outreach teams that go and speak to other doctors and nurses and talk about their experiences in the hospice, so that the doctors and nurses in hospital could maybe deal with it better.[P8 p35]

I don't know that we need even more meetings. I think the key thing is that it's always more effective to go in and talk to an existing group, such as a toddler group or bridge club, than to try get people to come into the hospice. [P5 p35]

There was agreement that the hospice should continue to host events and discussions; however, from experience these have not been well attended.

\section{Discussion}

This study, by its nature and objectives, was able to contribute a limited but insightful picture of a group discussion that explored the topic of talking about dying and death. Individuals in the community based group shared their views and perspectives very willingly; however, they were only a small sample who volunteered from a very large database of hundreds of names of people who had some sort of link with the local hospice. This was our first finding that in a sense our interest in talking about dying and death was not shared with many people in this community, and probably not many others as well. However, we had the opportunity to explore the issue of reluctance or disinterest with the study group.

In general, it seems that when people talk about discussing dying and death, the conversation bridges at least two, probably three distinct areas. There is a definite difference between planning how one wishes to spend their last days of life, and detailing the practicalities surrounding planning a funeral (i.e., location, music, who to contact, how to pay), or the more existential issues of life and death and our place on Earth.

This study set out to address three specific objectives that were of interest to the local hospice and funding body for this research, including: exploring perceptions of the local community, identifying barriers that may impede open discussion and proposing ways to increase participation. All these were achieved by active engagement with the public in discussion and by listening to their views and perceptions. Indirectly, this was planned as a way of instigating and promoting services that will be responsive to the needs of the local culture.

In terms of Emotions, Beliefs and Behaviours, the participants expressed their personal fears and the context of worry and acceptance of death. There was some notion of superstition, but little mention of formal religious beliefs. This is in contrast to an informative study by Dezutter and colleagues ${ }^{9}$ who examined the relation between religious attitudes and death attitudes. The findings from a questionnaire revealed that it is important to take a multidimensional approach to dying and death. For example, some people view death as final, others as a natural end-point, others view death as threatening and hostile, while still others believe death serves to give life meaning. Dezutter and colleagues ${ }^{9}$ found that openness to religion and transcendence gives a person a stronger belief in an after-life, however this is filtered through the individual's own level of defensiveness towards death (p. 90). ${ }^{9}$ Nevertheless, based upon the data available, the researchers argued that irrespective of whether people adopt a religious worldview or not, a literal, closed-minded and dogmatic approach to religious contents was associated with more death anxiety and with a stronger tendency to avoid and suppress death-related thoughts (p. 90]. ${ }^{9}$ This is a strong conclusion based on limited data and may be worthy of further investigation. In the meantime, the impact of religious belief on death anxiety is an interesting observation and could be considered in discussions on death and dying with patients, service users and families.

Within the themes, Coping with Adversity and Difficulties, Barriers and Tensions, lay the core of the community's views and needs. The link to the local hospice service was considered significant for it was where practical help, spiritual care and an appreciation for sensitivity, openness and honesty could be put into action. The importance of communication and language was found to be critical above all else. Concurring evidence was put forward in a comparative report by Russell and colleagues ${ }^{10}$ who invited individuals, families, professionals and communities to talk about death and dying. It was noted people who were dying and their loved ones wanted honest communication, 
[and] they appreciated health care practitioners (HCP) who were compassionate and truthful (p. 4). ${ }^{10}$ Talking and listening for HCPs, patients and their families were viewed as being essential in enabling people to think and thus talk about their own death or death and dying in general (pp. 11-16). ${ }^{10}$

Furthermore, according to an earlier study by Steinhauser et al., ${ }^{11}$ all direct discussions with staff, patients and family members, as opposed to avoidance techniques, were reported to assist and enable new ways in which to deal with the situations faced and in relationships. The authors acknowledged that within medical culture, death was often perceived as the negative outcome in the fight against disease, to discuss death, we fear, is to remove hope (p. 728). ${ }^{1}$ Avoidance was associated with increased suffering.

Data from the Steinhauser et al.'s study were gathered via focus groups and a survey questionnaire and add substance to support the importance of talking about dying and death. The participants reported that by engaging in discussions and preparing for the end of life they felt a sense of completion or closure. Some components of preparation deserved further discussion and these were: knowing the timing of death and discussing personal fears about dying. With the former, the physicians rated this as less important whereas the families and patients rated this highly. The latter was considered important by the families as opposed to patients. Regardless, both focus groups felt that the opportunity to prepare, in all its dimensions, for the end of life was preferential. Having decisions written down, knowing about what to expect from their physical condition and patients knowing that somehow their families were prepared, was important. Integrated dying - the tying up of loose ends, finishing unresolved business, remembering personal accomplishments and saying goodbye to important people was rated highly by the patients.
The last theme, Fostering a Participative Future brought out some suggestions of how the hospice could engage more with the local community in terms of improving understanding, communication and education on issues surrounding death. Any idea will have to depend on individuals to take them forward along with input and agreement from the local hospice. This is considered an area for discussion that is best left within the community itself and is probably not best served by academic criticism or debate.

\section{Conclusions}

In the context of our study, the focus group approach achieved its modest objectives of drawing out the perspectives of a small group of individuals who were willing to spend an evening discussing a topic that was of interest to them. They were not particularly unique, and the authors make no claim of the representativeness of the sample. However, it is through the honest speaking words and insights of these participants, that researchers, health care practitioners and managers in the field can continue to promote greater public service and awareness of death and dying issues. Further research on the role of health care professionals in talking about death and dying will be required. In addition, the cultural, ethnic and international dimensions would also be worthy of exploration and have practical implications for the development of more receptive, responsive and sensitive end-of-life care.

\section{References}

1. Department of Health. The End of Life Care Strategy: Second Annual Report. London:
Crown; 2010. Available from: http:/www.dh. gov.uk/publications

2. Locke, K. Let's Talk about death, say charities 2010. The Guardian. Accessed online: 13 January 2011.

3. Gallagher R. Using a Trade-Show format to educate the public about death and survey public knowledge and needs about issues surrounding death and dying. J Pain Symptom Manage 2001;21:52-8.

4. Burgess TA, Brooksbank M, Beilby JJ. Talking to patients death and dying. Aust Fam Physician 2004;33:85-6.

5. Dying Matter Coalition. GP Pilot Project Evaluation 2010. http:/www.dyingmatters. org/page/gp-pilot-project Accessed: 12 July 2011.

6. Morgan DL. The Focus Group Guidebook. London: Sage; 1998.

7. Speziale HJS, Carpenter DR. Qualitative Research in Nursing, Advancing the Humanistic Perspective (4th edn). Philadelphia: Lippincott Williams \& Wilkins; 2007.

8. Ritchie J, Spencer E. Qualitative data analysis for applied policy research. In, Bryman A, Burgess RG, eds. Analyzing Qualitative Data. London: Routledge; 1994.

9. Dezutter J, Soenens B, Luyckx K, et al. The role of religion in death attitudes: distinguishing between religious belief and style of processing religious contents. Death Studies 2009;33:73-92.

10. Russell S, Browne J, L'Estrange S. Talking about Dying: where and how we choose to die. Australia: Research Matters; 2004. Available from: http://www.researchmatters.net

11. Steinhauser KE, Christakis NA, Clipp EC, et al. Preparing for the end of life: preferences of Patients, families, physicians and other care providers. J Pain Symptom Manage 2001;22:727-37. 